\title{
Fore and Aft Channel Reconstruction in the TerraSAR-X Dual Receive Antenna Mode
}

\author{
Martina Gabele, Benjamin Bräutigam, Daniel Schulze, Ulrich Steinbrecher, \\ Nuria Tous-Ramon, and Marwan Younis, Senior Member, IEEE
}

\begin{abstract}
The TerraSAR-X satellite is a high-resolution synthetic aperture radar (SAR) system launched in June 2007 which provides the option to split the antenna in along-track direction and sample two physical channels separately. Modern SARs are equipped with active phased array antennas and multiple channels. In order to keep costs low, TerraSAR-X uses the redundant receiver unit for the second channel such that fore and aft channel signals are combined by a hybrid coupler to form sum and difference channel data. The dual receive antenna (DRA) mode can either be used to acquire along-track interferometric data or to acquire signals with different polarizations at the same time (Quad-Pol). Fore and aft channel reconstruction is necessary if ground moving target indication (GMTI) algorithms such as the displaced phase center antenna technique or along-track interferometry shall be applied, and in order to separate the horizontally and vertically polarized received signal components. The proposed approach uses internal calibration pulses from different calibration beams in order to estimate and compensate the hardware impact. The theoretical framework together with the results from the experimental data evaluation for the fore and aft channel reconstruction of the TerraSAR-X DRA mode are presented. The impact of the receive hardware transformation matrix estimation accuracy on errors in the reconstructed fore and aft channel image data is studied, and first examples on the GMTI capability of the TerraSAR-X DRA mode are given.
\end{abstract}

Index Terms-Along-track interferometry (ATI), calibration, dual receive antenna (DRA) mode, ground moving target indication (GMTI), space-based radar, synthetic aperture radar (SAR), TerraSAR-X.

\section{INTRODUCTION}

$\mathbf{T}$ HE TECHNOLOGICAL progress in remote sensing applications based on spaceborne synthetic aperture radar (SAR) developed flexible radar systems to satisfy the user needs for global Earth observation and reconnaissance. Recent satellite SAR instruments such as the German satellite TerraSAR-X [1], [2], the Canadian SAR satellite Radarsat-2 [3], and the Italian satellite constellation COSMO Sky-Med [4] are featuring multiple channels separated in along-track direction in order to not only produce high-resolution images of the Earth but also detect movements. Example applications for ground moving target indication (GMTI) are detection of road traffic [5], ship surveillance [6], river current measurements [7], [8], or oceanography [9], to name a few. Furthermore,

Manuscript received March 4, 2009; revised June 18, 2009, and September 7, 2009. First published November 13, 2009; current version published January 20, 2010.

The authors are with the Microwaves and Radar Institute, German Aerospace Center (DLR), 82234 Wessling, Germany (e-mail: Martina.Gabele@dlr.de; Benjamin.Braeutigam@dlr.de; Daniel.Schulze@dlr.de; Ulrich.Steinbrecher@ dlr.de; Nuria.TousRamon@dlr.de; Marwan.Younis@dlr.de).

Digital Object Identifier 10.1109/TGRS.2009.2032920 multiple channels separated in along-track direction allows for receiving different polarizations at the same time (in the socalled Quad-Pol mode). Applications thereof are in the field of polarimetry such as soil moisture or biomass estimation [10]. With TerraSAR-X, two receive channels separated in along-track direction can be realized either by switching between the fore and aft antenna halves from pulse to pulse, or with the dual receive antenna (DRA) mode. While the toggling in the single-channel switched aperture mode is implemented by alternately attenuating the fore and aft antenna halves, in the DRA mode, the signals of the fore and aft antenna halves are combined by a hybrid coupler [11] generating the sum and difference channel data. While the sum channel data are sampled by the main receiver unit, the difference channel data are sampled by the redundant receiver unit. Generating the sum and difference channel data instead of the fore and aft channel data is a low-cost and efficient method to implement a two-channel mode that requires little change to the front-end hardware at the cost of a more complex calibration procedure. In the along-track interferometric mode (ATI mode), fore and aft channel reconstruction in the processing is necessary, if GMTI algorithms such as the displaced phase center antenna (DPCA) technique or along-track interferometry (ATI) shall be applied [5], [12]. For the Quad-Pol mode, fore and aft channel reconstruction is necessary in order to separate the horizontally and vertically polarized received signal components, because one polarization is received by the fore, the other polarization by the aft channel. Although this paper focuses on the fore and aft channel reconstruction of the ATI mode, it is expected that the same channel reconstruction procedure is valid for the Quad-Pol mode as well, since both modes use the same transmit and receive hardware.

This paper is structured as follows. In Section II, the theoretical framework for the proposed approach of fore and aft channel reconstruction based on internal calibration pulses and calibration beams is derived [13]. Therefore, in Section II-A, the impact of the DRA mode transmit and receive hardware on the fore and aft channel imaging signals is explained. In Section II-B, the available calibration pulses are introduced and which hardware sections they characterize is explained. In Section II-C, the calibration beams available for the DRA mode data takes are introduced. With this understanding, a strategy for transformation matrix estimation, fore and aft channel reconstruction, and hardware impact compensation is introduced in Section II-E. Since a beneficial domain for the SAR image data analysis from along-track arrays is the range/Doppler domain, in Section III, the fore and aft channel image data as 


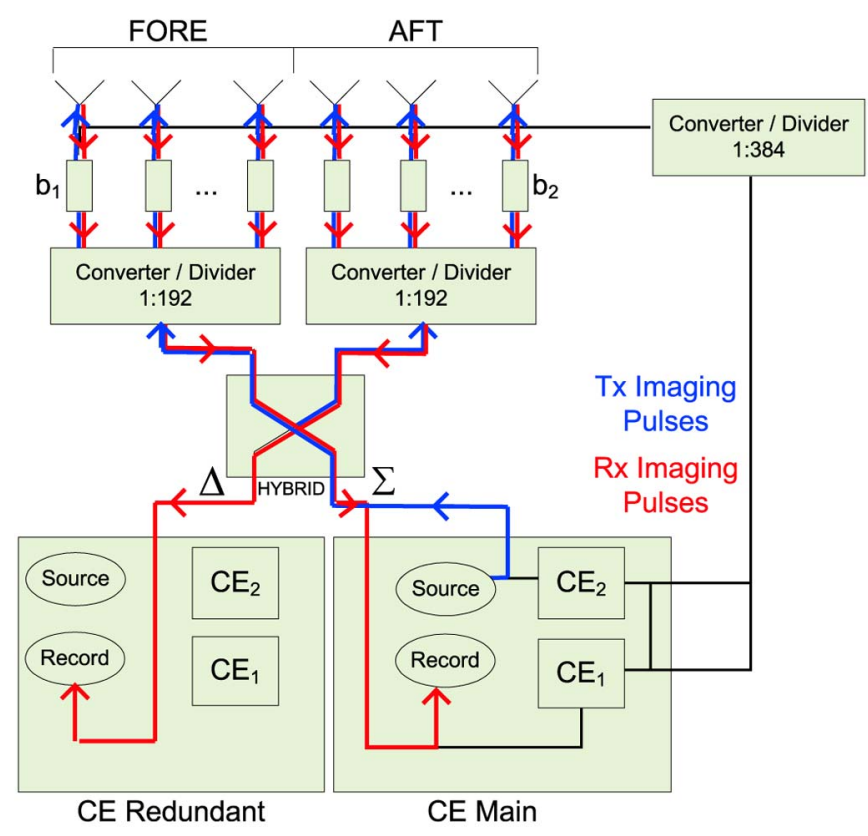

Fig. 1. Block diagram of the TerraSAR-X DRA mode hardware implementation. Paths of the imaging pulses (blue) on transmit and (red) on receive.

they appear at the fore and aft antenna halves are modeled in the range/Doppler domain. The corresponding expressions for the image data after receive hardware transformation in sum and difference channel domain are formulated in Section III-B. Based on this the propagation of errors in the receive hardware transformation matrix estimation to the reconstructed fore and aft channel imaging signals and the ATI signal is derived in Section III-C.

In Section IV, the results of the experimental data evaluation are presented. In Section IV-A, the two DRA mode campaigns that were performed in 2008 are briefly summarized and the TerraSAR-X system parameters as well as the data take parameters for the data takes analyzed in this paper are given. Analysis of the experimental imaging data is shown in Section IV-B. While the analysis of the imaging data gives first hints on the actual receive hardware transformation matrix, the complete transformation matrix can be estimated by use of the calibration pulses with different calibration beams. The analysis of the experimental data of the calibration pulses and the transformation matrix estimation is presented in Section IV-C. A first example on the GMTI capability of the TerraSAR-X ATI mode is given in Section IV-D. Section V summarizes the findings.

\section{Fore AND Aft ChANNEL RECONSTRUCTION}

A simplified hardware block diagram of the TerraSAR-X DRA mode realization is shown in Fig. 1. TerraSAR-X is a design-to-cost system, where the nominal SAR hardware is designed and optimized for single-channel operation, thus GMTI or fully polarimetric capabilities are not foreseen in nominal operation. However, the satellite includes redundant hardware for the critical components. The redundancy concept is such that two receive channels are available when both the nominal and the redundant hardware are used. Although this is against the common redundancy system usage strategy, it also offers the attractive possibility of using the aforementioned applications in special cases. Specifically two identical, instead of one, central electronics (CE) units are available, where the transmit and receive signals have to be routed such that any one of the CE units can be activated. If the two receive channels shall furthermore be usable for experimental two channel modes, this raises the question of how to route the hardware components. An elegant solution to this problem is the use of a hybrid junction: By the use of the hybrid junction the signals received by the whole antenna may be processed by the main $\mathrm{CE}$ as a sum beam if the phase shifts applied to the signals received by each of the antenna panels are such that they add up in phase for the main pointing direction. If, additionally, the redundant $\mathrm{CE}$ is used, the difference beam can as well be recorded. By transforming the sum and difference channel data back to fore and aft channel data, the original two channel data can be reconstructed. In case of failure of the main CE, the system can still be operated as a single-channel system by use of the redundant $\mathrm{CE}$ and application of a $\pi$ phase shift to the antenna panels of one antenna half, because this effectively turns the difference beam into a sum beam. It is this routing solution which requires an extra effort for fore and aft channel reconstruction.

The hardware characterization showed some fast frequency dependence as well as small variations from data take to data take. Furthermore, the phase between the sum and difference channel is not constant but rather changes from data take to data take and also as a function of fast frequency. One reason for this is the temperature change of the radio-frequency (RF) components used for signal routing. Furthermore, although only one ultrastable oscillator is used as reference for both CEs, each CE uses its own phase-locked loop (PLL) to generate the RF signal. In summary, both the cables' temperatures and the PLL result in small variations of the hardware, which can be kept constant during one data take, but vary from data take to data take. However, for spaceborne radar systems a high degree of agreement between the channels is required, which can be achieved by calibration. This is the case because due to the large separation between radar and target already small errors in the along-track interferometric phase cause for the example of GMTI a large target azimuth positioning error. Furthermore, the high platform velocity causes small along-track interferometric phases compared to airborne radar systems. For the example of GMTI, small phase errors transform into comparatively large errors in the estimated target radial velocity. For these reasons, the on-ground characterization of the receive hardware is not sufficient for the fore and aft channel reconstruction in the DRA mode. Instead, the receive hardware transformation matrix has to be estimated data-adaptively for each data take. The methodology for estimating the hardware transformation matrix is to measure the impact of the hardware on internal calibration pulses which travel through different sections of the hardware and to apply different calibration beams. In this section, the theoretical background is provided for an understanding of how hardware imperfections impact the imaging signals and the fore and aft channel signal reconstruction. A method for estimation and compensation of the hardware impact by use 
of calibration pulses and calibration beams is then developed. Considering this special hardware implementation this paper deals with a topic, which in this context is mostly relevant for TerraSAR-X. Nevertheless, considering the significance of the applications on one side and the fact that TanDEM-X (to be launched in 2009) will exploit the same redundancy technique, the presented solutions are considered of high relevance.

\section{A. Imaging Signals}

The paths of the imaging signals through the TerraSAR-X DRA mode hardware are shown in Fig. 1. The transmitted imaging signals (indicated in blue) are generated in the main channel and travel through the hybrid coupler from the main channel to the fore and aft channel transmit hardware where they are radiated by the antenna. The phased array antenna consists of 12 panels in azimuth. Each panel consists again of 32 rows in height. Together they make up 384 subelements, where each subelement is equipped with a transmit/receive module (TRM). On receive the antenna is split into fore and aft antenna halves. Behind the antenna, the received imaging signals (indicated in red) travel through the fore and aft receive hardware and are combined to form sum and difference channel signals in the hybrid coupler. Note that the antenna transmits as a single unit (where only one transmit polarization is possible at a time), but is split on receive. Behind the hybrid coupler, the signals travel on the main and redundant receive hardware until they are sampled and recorded. The 2-D fastfrequency/Doppler-frequency spectra of the received sum and difference channel imaging pulses $Z_{I, \Sigma}\left(f_{r}, f\right), Z_{I, \Delta}\left(f_{r}, f\right)$ with subscripts ${ }_{I}$ indicating imaging pulses are

$$
\left[\begin{array}{c}
Z_{I, \Sigma}\left(f_{r}, f\right) \\
Z_{I, \Delta}\left(f_{r}, f\right)
\end{array}\right]=\mathbf{H}_{\mathrm{Rx}}\left(f_{r}\right) H_{\mathrm{Tx}}\left(f_{r}\right)\left[\begin{array}{l}
S_{I, 1}\left(f_{r}, f\right) \\
S_{I, 2}\left(f_{r}, f\right)
\end{array}\right]+\left[\begin{array}{c}
N_{\Sigma} \\
N_{\Delta}
\end{array}\right]
$$

$$
\mathbf{H}_{\mathrm{Rx}}\left(f_{r}\right)=\left[\begin{array}{ll}
H_{11}\left(f_{r}\right) & H_{12}\left(f_{r}\right) \\
H_{21}\left(f_{r}\right) & H_{22}\left(f_{r}\right)
\end{array}\right]
$$

where the transfer function $H_{\mathrm{Tx}}\left(f_{r}\right)$ describes the whole transmit hardware path and $\mathbf{H}_{\mathrm{Rx}}\left(f_{r}\right)$ the whole receive hardware path as a function of the fast frequency or range frequency $f_{r}$. For the ideal receive hardware, $H_{11}\left(f_{r}\right)=H_{12}\left(f_{r}\right)=$ $H_{21}\left(f_{r}\right)=-H_{22}\left(f_{r}\right)=1 / \sqrt{2}$. The slow frequency or azimuth frequency dependence is expressed by $f . N_{\Sigma}$ and $N_{\Delta}$ are the additive white Gaussian noise (AWGN) components. The 2-D frequency spectra of the fore and aft channel signals $S_{I, 1}\left(f_{r}, f\right), S_{I, 2}\left(f_{r}, f\right)$ after SAR integration and azimuth compression [14], [15] can be expressed as follows:

$$
\begin{aligned}
& S_{I, 1}\left(f_{r}, f\right)=A(f) \cdot e^{j \frac{\pi d}{2 v} f} \cdot e^{-j \frac{\pi}{k_{r}} f_{r}^{2}} \\
& S_{I, 2}\left(f_{r}, f\right)=A(f) \cdot e^{-j \frac{\pi d}{2 v} f} \cdot e^{-j \frac{\pi}{k_{r}} f_{r}^{2}} .
\end{aligned}
$$

Subscript $_{1}$ indicates fore and subscript ${ }_{2}$ aft channel, $A(f)$ is the azimuth antenna pattern weighting versus Doppler, $d$ is the fore/aft along-track baseline, $v$ the inertial platform velocity, and $k_{r}=B / T$ the pulse chirp rate defined by the chirp bandwidth $B$ and the pulse duration $T$. For simplicity (and because it can be corrected in principal), range cell migration was neglected here. Furthermore, the pulse envelope was neglected.

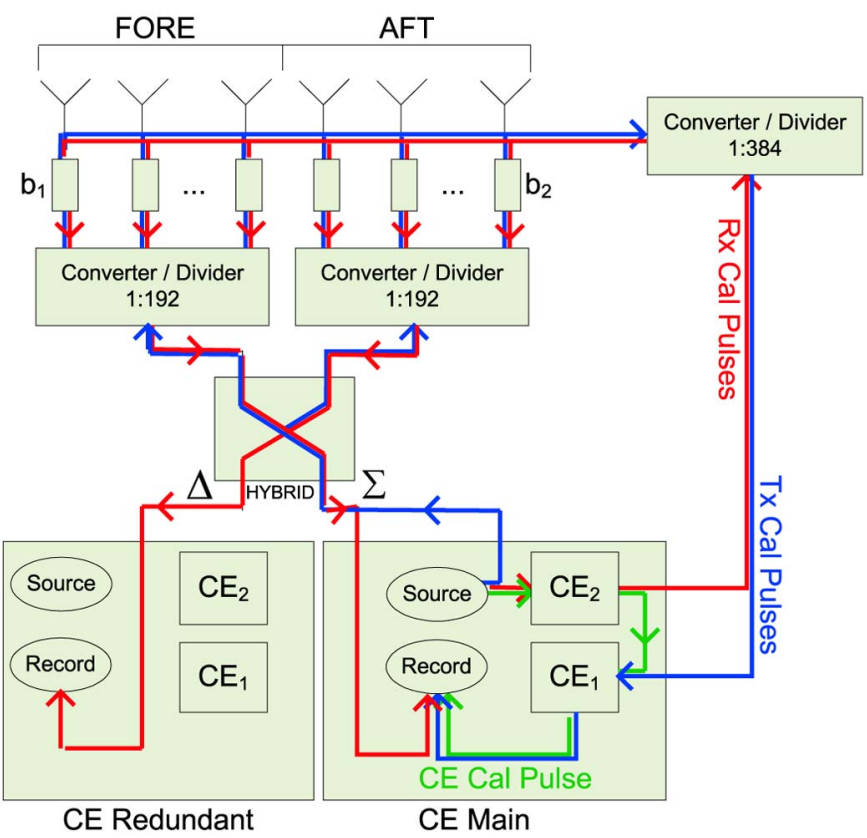

Fig. 2. Block diagram of the TerraSAR-X DRA mode hardware implementation. Paths of the (blue) Tx, (red) Rx, and (green) CE calibration pulses through the hardware.

Instead a perfectly rectangular pulse envelope was assumed and the pulse chirp is defined only for the fast-frequency interval where the rectangular pulse envelope is different from zero. The azimuth chirp can be assumed to be compensated (e.g., in the azimuth compression step of the SAR focusing). Furthermore, it does not play a role for the analysis of the interferometric signals since it cancels out.

\section{B. Calibration Pulses}

Calibration pulses are generated before, in between, and after each data take in order to calibrate the system [13], [16]. There are three types of calibration pulses which differ in the section of the hardware they characterize (Fig. 2): The transmit (Tx) calibration pulses (indicated in blue in Fig. 2) are sent through the transmit hardware of the system and are the same for both channels. Before the signals enter the antenna, they are coupled to the calibration network, which is only used to acquire the calibration pulses but which is not passed by the imaging pulses. This is the section starting behind the TRMs and ending where the Tx calibration pulses are recorded. The receive $(\mathrm{Rx})$ calibration pulses (indicated in red in Fig. 2) are sent through the receive hardware and characterize the fore and aft channel receive hardware between antenna and hybrid coupler, the hybrid coupler, as well as the section between hybrid coupler and the recording units. Similar to the Tx calibration pulses the Rx calibration pulses travel through some additional hardware, which is only used to acquire the calibration pulses but not passed by the imaging pulses. For the Rx calibration pulses, this is the hardware section starting behind the source, where the calibration pulses are generated until the Rx calibration pulses are fed into the TRMs. The third type of calibration pulses are the CE calibration pulses (indicated in green in Fig. 2) used to characterize the hardware sections, where only the Tx and Rx 
calibration pulses, but not the image data, pass through. While both $\mathrm{Tx}$ and both CE calibration pulses are pretty much the same for sum and difference channels, because they run through the same paths (except for a short hardware section which is not characterized), this is not true for the Rx calibration pulses: Fore and aft channel signals travel through the hardware of the fore and aft channel receive path before the hybrid coupler. In the hybrid coupler, the signals are combined to form sum and difference channel, and after the hybrid coupler the sum and difference channel signals travel on different propagation paths again.

As well as for the imaging pulses, the transmitted calibration pulses are modeled as ideal signals and the recorded calibration pulses $Z\left(f_{r}\right)$ as the transformed pulses. The transmitted Tx and $\mathrm{CE}$ calibration pulses can be expressed as

$$
S_{\mathrm{Tx}}\left(f_{r}\right)=S_{\mathrm{CE}}\left(f_{r}\right)=e^{-j \frac{\pi}{k_{r}} f_{r}^{2}}
$$

with amplitudes normalized to one and $f_{r}$ extending over the fast-frequency interval defined by the pulse chirp bandwidth $B$. For the Rx calibration pulses $S_{\mathrm{Rx}, n}\left(f_{r}\right), n=1,2$ describing the two physical channels of TerraSAR-X, where one stands for the fore channel and two represents the aft channel, various different complex weighting factors $b_{n}$, called calibration beams, can be applied

$$
S_{\mathrm{Rx}, n}\left(f_{r}\right)=b_{n} e^{-j \frac{\pi}{k_{r}} f_{r}^{2}}
$$

where subscript $n=1$ indicates fore and $n=2$ aft channel signals and $b_{n}$ is the sum of the weighting factors from 192 TRMs. By application of different weighting factors $b_{n}$, different calibration beams can be generated as is explained in Section II-C.

\section{Calibration Beams}

Calibration beams are complex amplitude weightings generated by the TRMs which are applied to the calibration pulses in order to study the hardware impact on different signals. The beams used here for the estimation of the receive hardware transformation matrix are the following.

- CalDRA: This calibration beam is the beam which is chosen for standard DRA mode data takes. Here, the calibration pulses sent through the fore channel receive hardware of the system are phase shifted by $b_{1}=e^{j \pi / 4}$, while the pulses sent through the aft channel receive hardware are phase shifted by $b_{2}=e^{-j \pi / 4}$. Due to the $\pi / 2$ phase offset, the sum and difference channel signals are expected to be of equal amplitudes.

- FORE: For this calibration beam, no phase offset is applied to the pulses sent through the fore and aft channel receive hardware. Instead, an amplitude weighting of $-20 \mathrm{~dB}$ is applied to the pulse which is sent through the aft channel receive hardware $\left(b_{1}=1, b_{2}=0.1\right)$.

Data takes with two kinds of calibration sequences are available. For the standard DRA mode data takes, only calibration pulses with the CalDRA beam were employed; for the DRA mode data takes with special calibration sequence, FORE and CalDRA calibration beams were employed.

\section{Transformation of Fore/Aft Channel Calibration Pulses}

The radar system can be assumed linear and time invariant here at least within one data take, where the time invariance is ensured by the temperature compensation mode [16], [17]. Therefore, the system can be characterized by transfer functions [18]. The transformation of the transmitted calibration pulses is analyzed in fast-frequency domain. The spectra of the Tx and $\mathrm{CE}$ calibration pulses recorded at the sum and difference channels $Z_{\mathrm{Tx}}\left(f_{r}\right)$ and $Z_{\mathrm{CE}}\left(f_{r}\right)$, can be written as

$$
\begin{aligned}
& Z_{\mathrm{Tx}}\left(f_{r}\right)=H_{\mathrm{CE}_{1}}\left(f_{r}\right) H_{\mathrm{Tx}}\left(f_{r}\right) S_{\mathrm{Tx}}\left(f_{r}\right)+N_{\mathrm{Tx}} \\
& Z_{\mathrm{CE}}\left(f_{r}\right)=H_{\mathrm{CE}_{1}}\left(f_{r}\right) H_{\mathrm{CE}_{2}}\left(f_{r}\right) S_{\mathrm{CE}}\left(f_{r}\right)+N_{\mathrm{CE}}
\end{aligned}
$$

with the scalar system transfer functions $H_{\mathrm{Tx}}\left(f_{r}\right)$ for the transmit hardware path of the imaging pulses; $H_{\mathrm{CE}_{1}}\left(f_{r}\right)$ for the hardware section that is used to acquire the Tx calibration pulses and that is characterized by the CE calibration pulses; and the AWGN components $N_{\mathrm{Tx}}$ and $N_{\mathrm{CE}}$. Accordingly, $H_{\mathrm{CE}_{2}}\left(f_{r}\right)$ is the transfer function of the hardware section used to acquire the Rx calibration pulses and is characterized by the CE calibration pulses. $S_{\mathrm{Tx}}\left(f_{r}\right)$ and $S_{\mathrm{CE}}\left(f_{r}\right)$ are the spectra of the transmitted Tx and CE calibration pulses defined in (4). The recorded sum and difference channel Rx calibration pulses, $Z_{\mathrm{Rx}, \Sigma}\left(f_{r}\right)$ and $Z_{\mathrm{Rx}, \Delta}\left(f_{r}\right)$, result in

$$
\begin{aligned}
& {\left[\begin{array}{l}
Z_{\mathrm{Rx}, \Sigma}\left(f_{r}\right) \\
Z_{\mathrm{Rx}, \Delta}\left(f_{r}\right)
\end{array}\right]=\mathbf{H}_{\mathrm{Rx}}\left(f_{r}\right) H_{\mathrm{CE}_{2}}\left(f_{r}\right) } \\
& \times\left[\begin{array}{l}
S_{\mathrm{Rx}, 1}\left(f_{r}\right) \\
S_{\mathrm{Rx}, 2}\left(f_{r}\right)
\end{array}\right]+\left[\begin{array}{l}
N_{\mathrm{Rx}, \Sigma}\left(f_{r}\right) \\
N_{\mathrm{Rx}, \Delta}\left(f_{r}\right)
\end{array}\right]
\end{aligned}
$$

where $S_{\mathrm{Rx}, 1}\left(f_{r}\right)$ and $S_{\mathrm{Rx}, 2}\left(f_{r}\right)$ are the spectra of the transmitted Rx calibration pulses and $N_{\mathrm{Rx}, \Sigma}$ and $N_{\mathrm{Rx}, \Delta}$ are the AWGN components.

Due to the fact that the phase reference of the sum and difference channel data is generated using two different local oscillators, the receive hardware transfer matrix $\mathbf{H}_{\mathrm{Rx}}\left(f_{r}\right)$ can in a first approximation be described as an ideal hybrid coupler fore/aft to sum/difference channel transformation followed by a phase offset $\Delta \nu$ between sum and difference channel

$$
\mathbf{H}_{\mathrm{Rx}}\left(f_{r}\right) \approx \frac{1}{\sqrt{2}}\left[\begin{array}{cc}
e^{j \Delta \nu} & e^{j \Delta \nu} \\
1 & -1
\end{array}\right] .
$$

The phase offset is stable during one data take, but varies between data takes. Two transformation matrix models are analyzed in the following: In the simple transformation matrix model, the receive hardware is assumed ideal and a varying phase offset between main and redundant channel is assumed. In the complete model, the receive hardware transformation matrix is estimated without applying any assumptions.

\section{E. Fore/Aft Channel Reconstruction Procedure}

The transformation matrix is estimated by use of the leastsquares method [19]. The objective of the least-squares method is to adjust the parameters of a model function to best fit a data set. In our case, the model function of the transformation $\mathbf{H}_{\mathrm{Rx}}\left(f_{r}\right) H_{\mathrm{CE}_{2}}(f r)$ of (8) is the transformation matrix $\hat{\mathbf{H}}_{\mathrm{Rx}}\left(f_{r}\right)$. The best fit is defined when the sum $e\left(f_{r}\right)$ of squared 
differences between the recorded calibration pulses and the calibration pulses predicted from the estimated model is minimum

$$
\begin{aligned}
e\left(f_{r}\right)= & {\left[Z_{\mathrm{Rx}, \Sigma, \text { Fore }}\left(f_{r}\right)-\left(\hat{H}_{11}\left(f_{r}\right)+0.1 \hat{H}_{12}\left(f_{r}\right)\right) e^{-j \frac{\pi}{k_{r}} f_{r}^{2}}\right]^{2} } \\
+ & {\left[Z_{\mathrm{Rx}, \Sigma, \text { CalDRA }}\left(f_{r}\right)\right.} \\
& \left.\quad-\left(e^{j \pi / 4} \hat{H}_{11}\left(f_{r}\right)+e^{-j \pi / 4} \hat{H}_{12}\left(f_{r}\right)\right) e^{-j \frac{\pi}{k_{r}} f_{r}^{2}}\right]^{2} \\
+ & {\left[Z_{\mathrm{Rx}, \Delta, \text { Fore }}\left(f_{r}\right)\right.} \\
& \left.\quad-\left(\hat{H}_{21}\left(f_{r}\right)+0.1 \hat{H}_{22}\left(f_{r}\right)\right) e^{-j \frac{\pi}{k_{r}} f_{r}^{2}}\right]^{2} \\
+ & {\left[Z_{\mathrm{Rx}, \Delta, \text { CalDRA }}\left(f_{r}\right)\right.} \\
& \left.\quad-\left(e^{j \pi / 4} \hat{H}_{21}\left(f_{r}\right)+e^{-j \pi / 4} \hat{H}_{22}\left(f_{r}\right)\right) e^{-j \frac{\pi}{k_{r}} f_{r}^{2}}\right]^{2} .
\end{aligned}
$$

The minimum of the sum of squares is found by setting the gradient to zero

$$
\begin{array}{lll}
\frac{\partial e\left(f_{r}\right)}{\partial \hat{H}_{11}\left(f_{r}\right)} \stackrel{!}{=} 0 & \frac{\partial e\left(f_{r}\right)}{\partial \hat{H}_{12}\left(f_{r}\right)} \stackrel{!}{=} 0 \\
\frac{\partial e\left(f_{r}\right)}{\partial \hat{H}_{21}\left(f_{r}\right)} \stackrel{!}{=} 0 & \frac{\partial e\left(f_{r}\right)}{\partial \hat{H}_{22}\left(f_{r}\right)} \stackrel{!}{=} 0 .
\end{array}
$$

Since the transformation matrix model contains four parameters, the gradient equations yield a system of four linear equations. This allows one to estimate $\hat{\mathbf{H}}_{\mathrm{Rx}}\left(f_{r}\right)$. The effect of $H_{\mathrm{CE}_{2}}\left(f_{r}\right)$ does not impact the quality of the fore and aft channel reconstruction. It would only have to be considered, if an absolute calibration is required. In this case, another calibration beam could be employed which allows one to establish a fifth equation in order to determine $H_{\mathrm{CE}_{2}}\left(f_{r}\right)$. Since some fastfrequency characteristics of the transformation matrix elements are expected, the estimation is performed as a function of fast frequency.

The ideal image data fore and aft channel spectra, $S_{I, 1}\left(f_{r}\right)$ and $S_{I, 2}\left(f_{r}\right)$ (with subscript ${ }_{I}$ indicating image data), are transformed by the transmit and receive hardware to form the sum and difference channel spectra $Z_{I, \Sigma}\left(f_{r}\right)$ and $Z_{I, \Delta}\left(f_{r}\right)$ according to (1). Therefore, after estimation of the receive hardware transformation matrix $\hat{\mathbf{H}}_{\mathrm{Rx}}\left(f_{r}\right)$ and fore and aft channel reconstruction, we obtain

$$
\left[\begin{array}{l}
Z_{I, 1}\left(f_{r}\right) \\
Z_{I, 2}\left(f_{r}\right)
\end{array}\right]=H_{\mathrm{Tx}}\left(f_{r}\right)\left[\begin{array}{l}
S_{I, 1}\left(f_{r}\right) \\
S_{I, 2}\left(f_{r}\right)
\end{array}\right]
$$

where $S_{I, 1}\left(f_{r}\right)$ and $S_{I, 2}\left(f_{r}\right)$ are the fast-frequency components of the ideal fore and aft channel imaging signals given in (2) and (3). $Z_{I, 1}\left(f_{r}\right)$ and $Z_{I, 2}\left(f_{r}\right)$ are the fast-frequency components of the fore and aft channel signals if $\hat{\mathbf{H}}_{\mathrm{Rx}}=\mathbf{H}_{\mathrm{Rx}}$. This means that the receive hardware impact has been removed, and with the replica signals [13], [20], solely the impact of the transmit hardware transfer function $H_{\mathrm{Tx}}\left(f_{r}\right)$ has to be compensated.

\section{IMAGe Data Modeling}

After reconstruction of the fore and aft channel data in ATI mode, compensation of the transmit and receive hardware impact and range compression, GMTI analysis techniques such as ATI, DPCA [5], [12], filtering with the inverse clutter covariance matrix, or approximations thereof [21], may be applied. A beneficial modeling and analysis of the reconstructed fore and aft channel SAR signals can be performed in range/Doppler domain. Since the fast-frequency dependence was compensated in the fore and aft channel reconstruction step, in the following, we neglect any fast-frequency dependence in (2) and (3). At first, the fore and aft channel imaging data in range/Doppler domain as they appear at the fore and aft antenna halves are modeled in Section III-A. Next, the sum and difference channel imaging signals after receive hardware transformation are expressed in Section III-B. Based on these two sections, an understanding for the shape of the reconstructed fore and aft channel signals depending on the back-transformation matrix used can be developed. The propagation of errors in the backtransformation matrix to the reconstructed fore and aft channel signals is expressed in Section III-C.

\section{A. Fore/Aft Channel Image Signals}

Assuming that the transmit and receive hardware impact was compensated in (2) and (3), the recorded fore and aft channel azimuth Doppler spectra $\mathbf{Z}_{I}(f)=\left[Z_{I, 1}(f), Z_{I, 2}(f)\right]$ of a single-point scatterer on the ground become

$$
\begin{aligned}
& Z_{I, 1}(f)=S_{I, 1}(f)+N_{1} \\
& Z_{I, 2}(f)=S_{I, 2}(f)+N_{2}
\end{aligned}
$$

with $N_{1}$ and $N_{2}$ being the AWGN components. Because azimuth ambiguities cannot be neglected for the TerraSAR$\mathrm{X}$ ATI mode, the modeling is extended to include the first-order azimuth ambiguities. More specifically, $\mathbf{S}_{I}(f)=$ $\left[S_{I, 1}(f), S_{I, 2}(f)\right]$ with $f \in[-P R F / 2, P R F / 2]$ is the vector containing the fore and aft channel signals in Nyquist band, while $\mathbf{S}_{I}(f+P R F)$ and $\mathbf{S}_{I}(f-P R F)$ is the vector containing the fore and aft channel first-order azimuth ambiguous signals, where $P R F$ is the pulse repetition frequency. Due to the long coherent integration time, as is typical for SAR systems, the Doppler bins decorrelate and the cross spectral density matrix of the clutter process $\mathbf{R}_{c, 1 / 2}(f)$ can be modeled as follows [22]:

$$
\begin{aligned}
\mathbf{R}_{c, 1 / 2}(f)= & E\left\{\mathbf{Z}_{I}(f) \mathbf{Z}_{I}^{\dagger}(f)\right\} \\
= & \sigma^{2} \sum_{m=-1}^{1} \mathbf{S}_{I}(f+m P R F) \\
& \times \mathbf{S}_{I}^{\dagger}(f+m P R F)+\mathbf{R}_{n}(f)
\end{aligned}
$$

where $\sigma^{2}$ is the received clutter power; ${ }^{\dagger}$ denotes conjugate complex transposed; and $\mathbf{R}_{n}(f)$ is the cross spectral density matrix of the AWGN. Since the AWGN is assumed to be temporally and spatially uncorrelated, $\mathbf{R}_{n}(f)$ is a diagonal matrix with the noise power $\sigma_{n}^{2}$ of each channel on the main diagonals. Concerning the estimation of the signals versus Doppler, it has to be noted that the interferometric phase of the stationary target Doppler spectrum is proportional to the sinus of the azimuth angle of arrival and independent of range [21]. Therefore, a 
very good signal quality can be achieved by averaging the interferometric signals in range/Doppler domain versus range.

\section{B. $\Sigma / \Delta$ Channel Image Signals}

If the fore/aft to sum/difference channel transformation induced by the $\mathrm{Rx}$ hardware section $\mathbf{H}_{\mathrm{Rx}}$ formulated in (1) is performed, the expression for the cross spectral density matrix $\mathbf{R}_{c, \Sigma / \Delta}(f)$ expands to

$$
\begin{aligned}
\mathbf{R}_{c, \Sigma / \Delta}(f)=\sigma^{2} \sum_{m=-1}^{1} & \mathbf{H}_{\mathrm{Rx}} \mathbf{S}_{I}(f+m P R F) \\
& \times \mathbf{S}_{I}^{\dagger}(f+m P R F) \mathbf{H}_{\mathrm{Rx}}^{\dagger}+\mathbf{R}_{\mathbf{n}}(f) .
\end{aligned}
$$

With no azimuth ambiguities, the phase of the interferometric signal in the fore and aft channel domain computed from (14) and (15) is $\angle(I(f)):=\angle\left(S_{I, 1}(f) \cdot S_{I, 2}^{\star}(f)\right)=$ $\pi d f / v$, where ${ }^{\star}$ denotes complex conjugate. Thus, $I(f)$ is a linear phase ramp over Doppler frequency. The sum and difference channel Doppler spectra of a single nonmoving point scatterer in case of ideal transmit and receive hardware $\mathbf{S}_{I}^{\prime}(f)=\left[S_{I, \Sigma}(f), S_{I, \Delta}(f)\right]^{\mathrm{T}}$ and negligible azimuth ambiguities are

$$
\begin{aligned}
& S_{I, \Sigma}(f)=\sqrt{2} A(f) \cos \left(\frac{\pi d}{2 v} f\right) \\
& S_{I, \Delta}(f)=j \sqrt{2} A(f) \sin \left(\frac{\pi d}{2 v} f\right) .
\end{aligned}
$$

The phase of the interferometric signal in sum and difference channel domain $\angle\left(I_{\Sigma / \Delta}(f)\right)=\angle\left(S_{\Sigma}(f) \cdot S_{\Delta}^{\star}(f)\right)$ yields a constant phase $\pi / 2$ for all negative Doppler frequencies with $-1<d \cdot f / v<0$ and a constant phase $-\pi / 2$ for all positive Doppler frequencies with $0<d \cdot f / v<1$. At zero Doppler, the phase jumps by $-\pi$.

The simulated azimuth Doppler spectra of the fore/aft channels and the sum/difference channels assuming ideal hybrid coupler transformation as given in (14), (15), (18), and (19) are shown in Fig. 3. The absolute values are shown in (a) and (c) while the ATI phases are shown in (b) and (d). The fore and aft channel signals have the maximum at zero Doppler and a linear ATI phase ramp. The sum channel signal has its maximum at zero Doppler as well, where the maximum value is increased by $3 \mathrm{~dB}$ compared to the fore and aft channel maxima. The difference channel has its minimum at zero Doppler and the sum/difference channel ATI phase is constant versus Doppler frequency with a phase jump of $-\pi$ at zero Doppler.

With no azimuth ambiguities, the first and second eigenvalues $\lambda_{1}(f), \lambda_{2}(f)$ of the fore/aft channel cross spectral density matrix versus Doppler defined in (17) can be found to be

$$
\begin{aligned}
& \lambda_{1}(f)=2 \sigma^{2}|A(f)|^{2}+\sigma_{n}^{2} \\
& \lambda_{2}(f)=\sigma_{n}^{2} .
\end{aligned}
$$

While the first eigenvalue represents the antenna pattern weighting versus Doppler that was contained in the fore and aft channel data (14), (15) increased by a gain factor of two for a two channel system, the second eigenvalue gives the noise level contained in the fore and aft channel signals. When

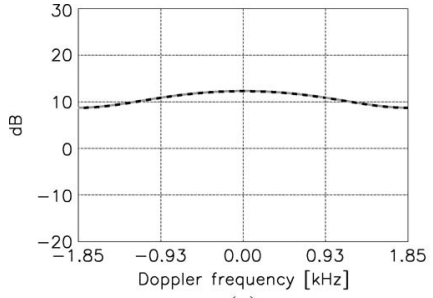

(a)

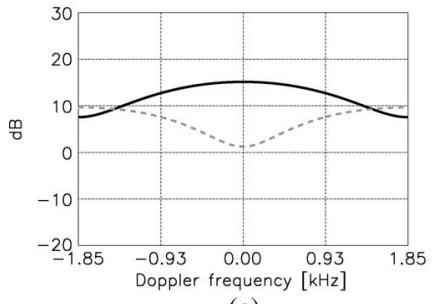

(c)

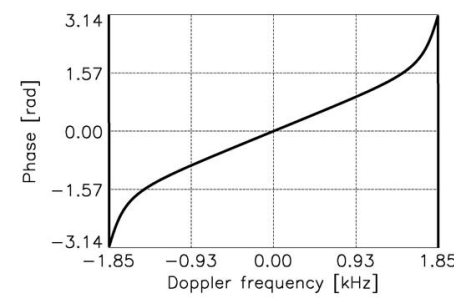

(b)

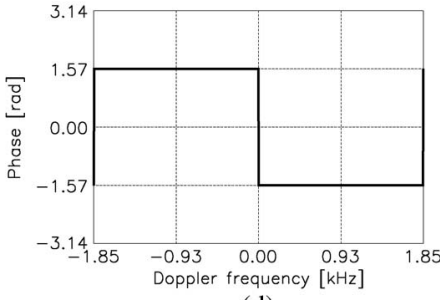

(d)
Fig. 3. (a) Fore/Aft amplitude. (b) Fore/Aft ATI phase. (c) Sum/Difference amplitude. (d) Sum/Difference ATI phase. (Dashed) Aft or difference channel.

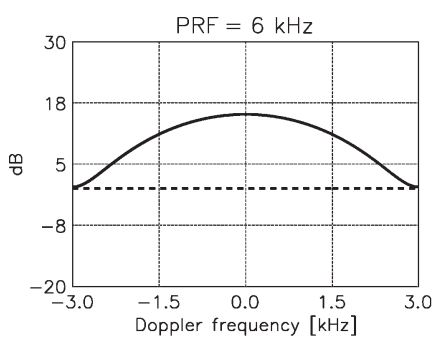

(a)

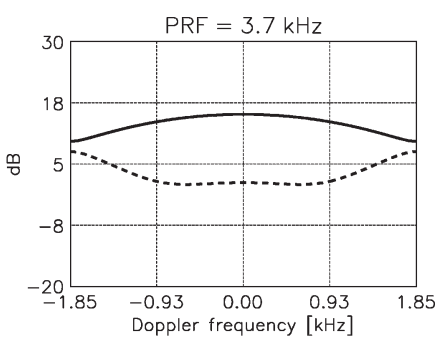

(b)
Fig. 4. Eigenvalue spectra for high and low $P R F$ (noise level normalized to $0 \mathrm{~dB}$ ). (a) High PRF. (b) Low PRF.

azimuth ambiguities have to be included in the modeling, essentially the second eigenvalue increases toward the edges of the Nyquist band.

In Fig. 4, the eigenvalue Doppler spectra are shown for two different $P R F s$. In (a), the $P R F$ was chosen such that azimuth ambiguities do not impact the eigenvalue spectra. In this case, the first eigenvalue (solid) shows the azimuth antenna pattern weighting of the fore, respectively, aft channel, increased by $3 \mathrm{~dB}$ according to (20), and the second eigenvalue (dashed) is at noise level (21). If the PRF is chosen such that azimuth ambigutities impact the eigenvalue Doppler spectra [Fig. 4(b)], the level of the second eigenvalue is increased toward the edges of the Nyquist spectrum.

With this knowledge about the expected fore and aft channel as well as sum and difference channel imaging data in mind, we can study the impact of the usage of different backtransformation matrices on the shape of the reconstructed fore and aft channel imaging data and the deviations from the ideally expected original fore and aft channel data as given in Section III-A. With the knowledge about the ideally expected sum and difference channel data in mind, we can get first hints on the actual transformation matrix by comparing the actual image data with the ideally expected sum and difference channel data as given in this section. This is important because for the DRA mode, variations in the receive hardware from data take to data take have been observed, and the characterization of the 
TerraSAR-X DRA mode hardware impact is necessary for each data take. Since the knowledge about the hardware is gained from calibration pulses, the accuracy of the knowledge depends on the signal to noise ratio $(S N R)$ and the number of calibration pulses employed for each calibration beam. Uncertainties in the knowledge about the receive hardware transformation matrix cause errors in the reconstructed fore and aft channel signals.

\section{Propagation of Errors in the $\Sigma / \Delta$ to Fore/Aft Transformation}

The propagation of errors in the estimation of $\mathbf{H}_{\mathrm{Rx}}$ to the reconstructed fore and aft channel imaging signals in case of an erroneous reconstruction can be formulated by applying the Gaussian error propagation law in complex arithmetic [23]. The reconstructed fore and aft channel signals, as well as any metric formed out of these signals such as the ATI phase, are influenced not only by the noise in the transformation matrix elements but also by the noise in the sum and difference channel data. As an example application, we formulate here the impact on the clutter interferometric signal versus Doppler frequency. The error propagation could however accordingly be formulated for moving target signals and GMTI metrics. The clutter interferometric signal $Z_{\text {ati }}(f)$ as a function of Doppler is

$$
\begin{aligned}
Z_{\text {ati }}(f)= & Z_{I, 1}(f) Z_{I, 2}^{\star}(f) \\
= & {\left[K_{11} Z_{I, \Sigma}(f)+K_{12} Z_{I, \Delta}(f)\right] } \\
& \cdot\left[K_{21} Z_{I, \Sigma}(f)+K_{22} Z_{I, \Delta}(f)\right]^{\star}
\end{aligned}
$$

where the reconstructed fore and aft channel imaging signals $Z_{I, 1}(f)$ and $Z_{I, 2}(f)$ are expressed in terms of the sum and difference channel imaging data $Z_{I, \Sigma}(f)$ and $Z_{I, \Delta}(f)$ and the elements $K_{i j}$ with $i=1,2$ and $j=1,2$ of the backtransformation matrix $\mathbf{H}_{\mathrm{Rx}}^{-1}$. Assuming that the input variables are pairwise uncorrelated and real and imaginary parts of each input variable are identically distributed and uncorrelated, the variance of the ATI signal versus Doppler frequency is

$$
\begin{aligned}
\sigma_{\mathrm{ati}}^{2}(f)=\sigma_{\Sigma}^{2}\left(\frac{\partial Z_{\mathrm{ati}}(f)}{\partial Z_{I, \Sigma}}\right)^{2} & +\sigma_{\Delta}^{2}\left(\frac{\partial Z_{\mathrm{ati}}(f)}{\partial Z_{I, \Delta}}\right)^{2} \\
& +\sum_{i=1}^{2} \sum_{j=1}^{2} \sigma_{K_{i j}}^{2}\left(\frac{\partial Z_{\mathrm{ati}}(f)}{\partial K_{i j}}\right)^{2}
\end{aligned}
$$

where $\sigma_{\Sigma}$ and $\sigma_{\Delta}$ are the standard deviations of the sum and difference channel signals; while $\sigma_{K_{i j}}$ are the standard deviations of the elements of the back-transformation matrix $\mathbf{H}_{\mathrm{Rx}}^{-1}$; and $\partial$ means partial derivative. In the following, the propagation of errors is simulated for the simple receive hardware transformation matrix model according to (9) and the complete model.

1) Simple Transformation Matrix Model: In the simple transformation matrix model, the phase offset between sum and difference channel data has to be estimated and compensated prior to fore/aft channel reconstruction. The impact of an uncompensated phase offset $\Delta \nu$ at the sum channel signal on the amplitude spectra versus Doppler frequency of the reconstructed fore and aft channel signals is shown in Fig. 5. An uncompensated sum channel phase offset $\Delta \nu$ causes the Doppler centroids of the reconstructed fore and aft channel sig-

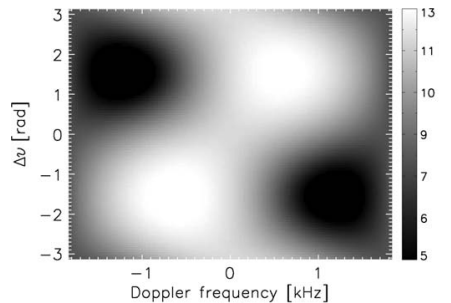

(a)

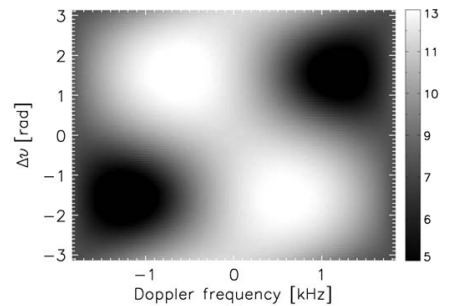

(b)
Fig. 5. Amplitude spectra (in $\mathrm{dB}$ ) versus Doppler frequency of reconstructed fore and aft channel signals as a function of an uncompensated sum channel phase offset $\Delta \nu(\Delta \nu=0$ is the ideal case). (a) Reconstructed fore channel. (b) Reconstructed aft channel.

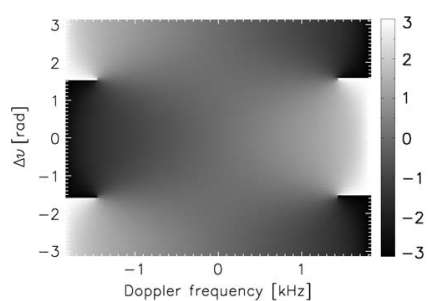

(a)

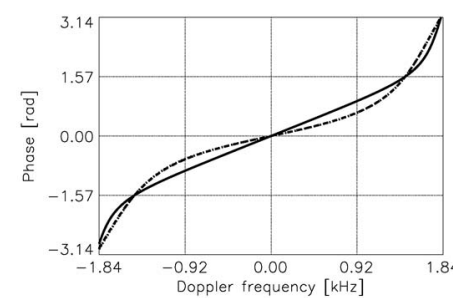

(b)
Fig. 6. (a) ATI phase (in radians) versus Doppler frequency of reconstructed fore and aft channel signals as a function of an uncompensated sum channel phase offset $\Delta \nu$. (b) Cuts through the ATI phase versus Doppler frequency plane on the left at (solid) $\Delta \nu=0 \mathrm{rad}$ and (dashed and dotted/dashed) $\Delta \nu= \pm 1 \mathrm{rad}$.

nals to disperse. Furthermore, as shown in Fig. 6, this changes the interferometric phase ramp versus Doppler frequency of the reconstructed fore and aft channel signals. For uncompensated phase offsets $|\Delta \nu|<\pi / 2$, the interferometric phase ramp becomes nonlinear and less steep around zero Doppler as is shown in Fig. 6 on the right.

2) Complete Transformation Matrix Model: The framework for a more precise study of the impact from uncertainties in the transformation matrix elements on the reconstructed fore and aft channel signals and their ATI signal provided in Section III-C allows one to study the impact of the image data $S N R$ and the back-transformation matrix elements' $S N R$ on the estimation of the along-track baseline that is the effective fore and aft channel phase center separation, which is an important aspect for GMTI applications [24]. In Fig. 7, the effective along-track baseline estimation out of the simulated image data is explained: The spatial displacement of the fore and aft antenna phase centers causes a time shift between the fore and aft channel image data. In Doppler frequency domain, this time shift transforms to an ATI phase ramp. Therefore, by estimation of the slope of the ATI phase ramp, one can determine the effective along-track baseline between the fore and aft channel data. In solid line, the true ATI phase ramp is shown, while the dashed tube around the true ATI phase ramp defines the limitation of the ATI phase fluctuations given by the standard deviations for a signal with $S N R=10 \mathrm{~dB}$ at the azimuth beam maximum. In order to avoid biases for the baseline estimation, a Doppler-frequency interval should be chosen which is free of azimuth ambiguities. We limit the frequency interval to $f \in$ $[-1 \mathrm{kHz}, 1 \mathrm{kHz}]$. The maximum and minimum possible baselines which could be estimated incorporating the uncertainties 


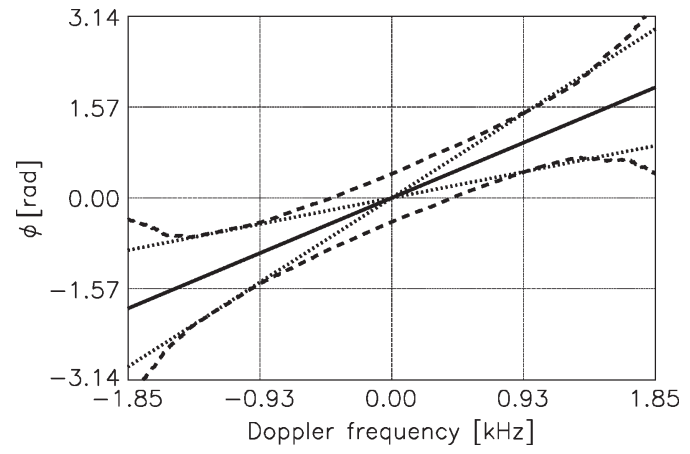

Fig. 7. Impact of image data $S N R$ on the ATI phase. (Solid) True fore/aft channel along-track interferometric phase. (Dashed) Tube around the expected interferometric phase ramp limited by the standard deviations of the ATI phases for a signal with $S N R=10 \mathrm{~dB}$ at azimuth pointing direction. (Dotted) Minimum and maximum baselines that could be estimated from the ATI phase signal if the signal in the Doppler-frequency interval $f \in[-1 \mathrm{kHz}, 1 \mathrm{kHz}]$ is considered.

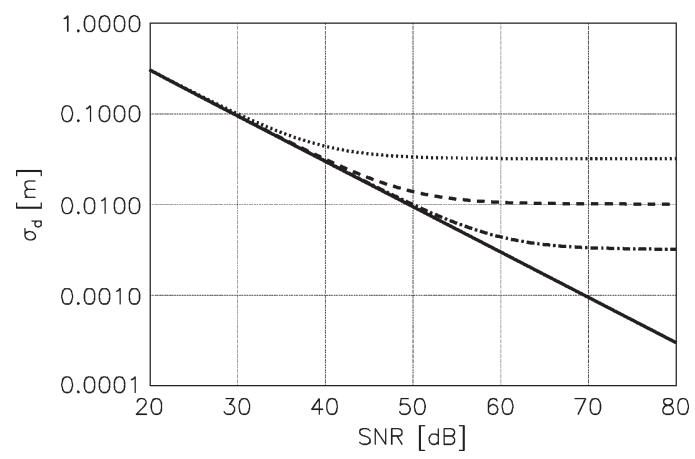

Fig. 8. Impact of image data $S N R$ and the transformation matrix elements' $S N R$ on the effective along-track baseline estimation accuracy $\sigma_{d}$. Shown is the standard deviation of the along-track baseline estimation using the fore/aft channel ATI phase ramp for $f \in[-1 \mathrm{kHz}, 1 \mathrm{kHz}]$ versus the image data $S N R$. (Solid) Ideally known back-transformation matrix elements. (Dotted) $S N R=$ $40 \mathrm{~dB}$ for back-transformation matrix elements. (Dashed) $S N R=50 \mathrm{~dB}$ for back-transformation matrix elements. (Dotted and dashed) $S N R=60 \mathrm{~dB}$ for back-transformation matrix elements.

of the ATI phase ramp are sketched in dotted lines. Perfectly known back-transformation matrix elements are assumed here. Uncertainties are only due to the image data $S N R$.

In Fig. 8, the standard deviation of the baseline estimation versus image data $S N R$ obtained from simulation using the baseline estimator described above for a number of different back-transformation matrix elements' $S N R$ is shown. In solid line, the standard deviation of the baseline estimation only due to image data $S N R$, but with perfectly known backtransformation matrix elements is shown. For example, the $S N R$ required to estimate the baseline with an uncertainty of $2 \sigma=1 \mathrm{~mm}$ is about $70 \mathrm{~dB}$. In case that the back-transformation matrix elements are known with limited accuracy, they determine the maximum possible baseline estimation accuracy. If for the back-transformation matrix elements $S N R=40 \mathrm{~dB}$, the maximum possible baseline estimation accuracy is $2 \sigma \approx 5 \mathrm{~cm}$ (dotted), for $S N R=50 \mathrm{~dB} 2 \sigma \approx 1.5 \mathrm{~cm}$ (dashed), and for $S N R=60 \mathrm{~dB} 2 \sigma \approx 5 \mathrm{~mm}$ (dashed and dotted). When looking at these $S N R$ values, one has to consider that the $S N R$ can be increased by $10 \log (N)$ by averaging versus $N$ samples. For the estimation of the back-transformation matrix elements, averaging versus the number of calibration pulses for each calibration beam can be performed. Additionally, one could average versus neighboring fast-frequency samples. However, it has to be taken into account that this reduces the fast-frequency resolution of the transformation matrix elements. For increasing the $S N R$ with the image data, one could perform averaging versus range and if the integration time is sufficiently long versus neighboring Doppler bins.

\section{EXPERIMENTAl DATA RESUlts}

The TerraSAR-X system parameters and the system parameters of the data takes analyzed in this paper are listed in Section IV-A. For the reconstruction of the fore and aft channel signals, two sources of information are available in order to study the hardware impact for this mode: The image data can be used to get hints on the actual hardware transformation matrix by comparison of the image data with the ideally expected image data as defined in Section III-B. However, usually, the image data are not used for a precise hardware characterization, since the image data may, for example, contain effects due to the inhomogeneity of the backscattering surface or other real world effects. The results of the image data analysis nevertheless are presented in Section IV-B. Precise hardware characterization is usually performed by use of internal calibration pulses and calibration beams. The results of the DRA mode hardware characterization using calibration pulses and calibration beams are presented in Section IV-C. A first example on the GMTI capability of the TerraSAR-X ATI mode is given in Section IV-D.

\section{A. DRA Mode Campaigns}

With TerraSAR-X so far, two DRA mode campaigns were performed, one in July and one in December 2008. During both campaigns, multiple data takes were acquired in ATI and in Quad-Pol mode with varying radar system parameters. The sensor operates at a center frequency of $9.65 \mathrm{GHz}$ with a maximum bandwidth of $300 \mathrm{MHz}$ and a maximum $P R F$ of $6750 \mathrm{~Hz}$. Relevant system parameters are summarized in Table I. The system parameters adopted for the data takes discussed in this paper are listed in Table II. FIDnum is the file identification number [25], the Date gives the day of the acquisition, while the Time is the universal time coordinated commanded start time of the data take acquisition, and $B$ is the pulse chirp bandwidth used. The last row shows the country over which the data take was acquired.

\section{B. Image Data Analysis}

In Fig. 9, on the top, the image data sum and difference channel amplitude and phase Doppler spectra after averaging versus range of an ATI mode data take acquired in July 2008 are shown, while on the bottom, the sum and difference channel amplitude and phase Doppler spectra of an ATI mode data take acquired in December 2008 are shown. Comparing these Doppler spectra with the Doppler spectra expected from Fig. 3, we notice that the amplitude spectra look very much the same for both data takes. Differences are mainly in the edges of 
TABLE I

TERRASAR-X System PARAMETERS

\begin{tabular}{|l|c|}
\hline Center Frequency & $9.65 \mathrm{GHz}$ \\
\hline Orbit altitude & $514 \mathrm{~km}$ \\
Platform velocity & $7.56 \mathrm{~km} / \mathrm{s}$ \\
Radiated RF Power & $2 \mathrm{~kW}$ (Peak) \\
Antenna Size & $4.8 \mathrm{~m}$ (azimuth), $0.7 \mathrm{~m}$ (elevation) \\
Bandwidth & $300 \mathrm{MHz}$ max. \\
$P R F$ & $6750 \mathrm{~Hz}$ max. \\
Polarization & $\mathrm{H}$ and $\mathrm{V}($ single, dual, quad) \\
Look Angle Range & $15^{\circ}-60^{\circ}$ \\
\hline Operation Modes & StripMap, ScanSAR, \\
& Sliding Spotlight \\
\hline
\end{tabular}

TABLE II

System PARAMETERS FOR DRA MOde DATA TAKES

\begin{tabular}{|l|c|c|c|}
\hline FIDnum & 8988 & 9613 & 4101 \\
\hline \hline Date & 17.07 .2008 & 28.07 .2008 & 11.12 .2008 \\
Time & $15: 34: 25$ & $17: 09: 59$ & $05: 16: 59$ \\
B & $100 \mathrm{MHz}$ & $150 \mathrm{MHz}$ & $300 \mathrm{MHz}$ \\
PRF & $3704 \mathrm{~Hz}$ & $3630 \mathrm{~Hz}$ & $6347 \mathrm{~Hz}$ \\
Country & Ukraine & Germany & Germany \\
\hline
\end{tabular}

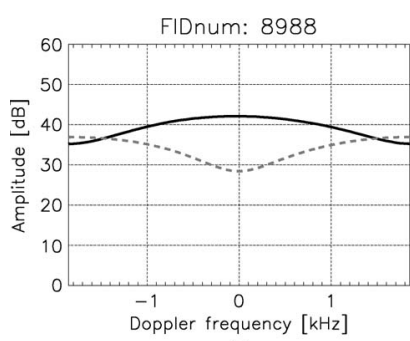

(a)

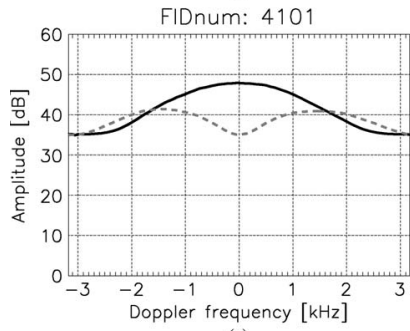

(c)

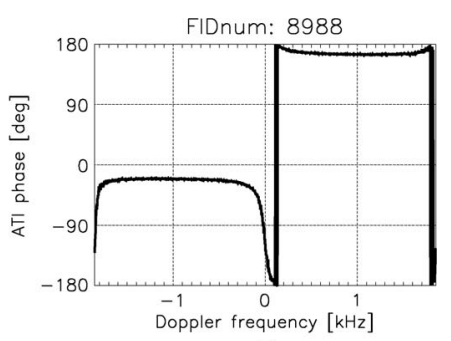

(b)

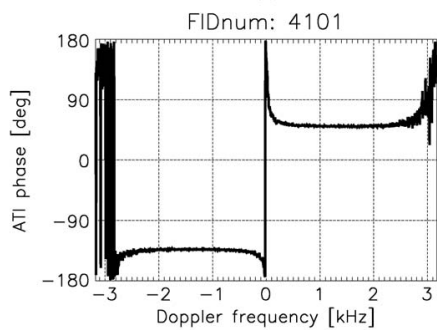

(d)
Fig. 9. (a) and (c) (Solid) Sum and (dashed) difference channel amplitude spectra versus Doppler frequency. (b) and (d) Interferometric phase versus Doppler frequency.

the Nyquist spectrum, where the impact of azimuth ambiguities can be seen for data take FIDnum $=8988$ due to the lower PRF. The interferometric phase spectra are essentially what we would expect from (18), (19), constant phases versus Doppler frequency with a phase jump of $-\pi$ at zero Doppler, but with a significantly different phase offset in both cases. In Fig. 10, the reconstructed fore and aft channel amplitude and interferometric phase spectra versus Doppler frequency are shown for a reconstruction with a constant sum channel phase offset estimated out of the CalDRA Rx calibration pulses just like in the case of the standard ATI mode data takes. This methodology refers to the simple transformation matrix model as is described in Section III-C1. As shown in Fig. 10, this yields the desired results. A more detailed characterization

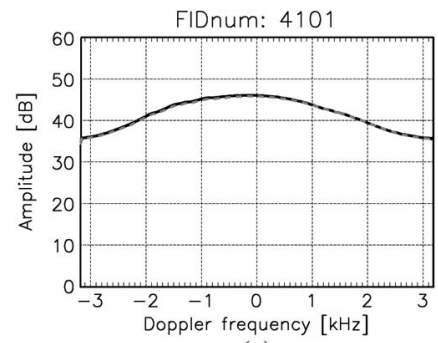

(a)

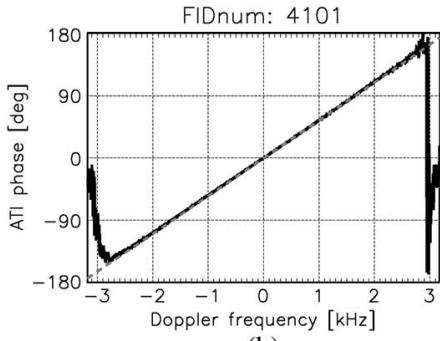

(b)
Fig. 10. (a) Reconstructed (solid) fore and (dashed) aft channel amplitude spectra versus Doppler frequency. (b) Interferometric phase versus Doppler frequency.

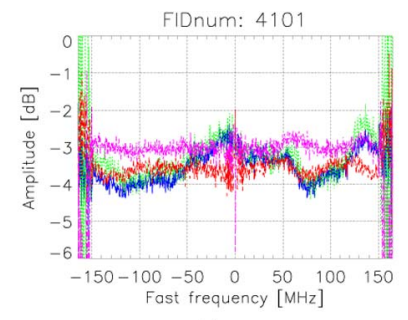

(a)

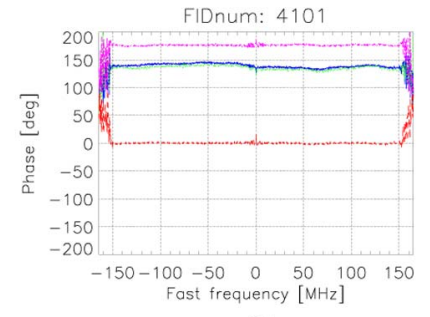

(b)
Fig. 11. (a) Amplitudes and (b) phases of the fore/aft to sum/difference channel transformation matrix elements. $H_{11}\left(f_{r}\right)$ : blue. $H_{12}\left(f_{r}\right)$ : green. $H_{21}\left(f_{r}\right)$ : red. $H_{22}\left(f_{r}\right)$ : magenta.

of the DRA mode hardware impact is, however, possible by analysis of the calibration pulses.

\section{Calibration Pulses Analysis}

As was outlined in Section II-E, a complete estimation of the receive hardware transformation matrix is possible if multiple calibration beams are applied. The estimated complete fore/aft to sum/difference channel transformation matrix elements according to Section II-E are shown in Fig. 11. As was expected from (9), the amplitudes of the transformation matrix elements are at about $-3 \mathrm{~dB}$; for the phases, we see the already mentioned phase offset $\Delta \nu$ in the elements $H_{11}\left(f_{r}\right), H_{12}\left(f_{r}\right)$, which define the transfer of the fore and aft channel signals to the sum channel port. $H_{21}\left(f_{r}\right)$ and $H_{22}\left(f_{r}\right)$ do not contain a significant phase offset, but have opposite signs, because these transfer functions define the transition of the fore and aft channel signals to the difference channel port. Some variation over frequency in amplitude and phase of the transformation matrix elements can, however, be noticed.

As was outlined in Section III-C2, for the example of the ATI phase versus Doppler, which may be used in order to exactly estimate the effective along-track baseline out of the imaging data in range/Doppler domain, is highly sensitive to errors in the estimated receive hardware transformation matrix. Therefore, an important aspect for the quality of the reconstructed fore and aft channel signals is the accuracy with which the reconstruction matrix is known. The estimated mean and standard deviations of the real and imaginary parts of the backtransformation matrix elements are shown in Fig. 12. For the data take, analyzed eight calibration pulses for each calibration beam were available. Averaging was performed only versus the number of calibration pulses, but not versus neighboring 


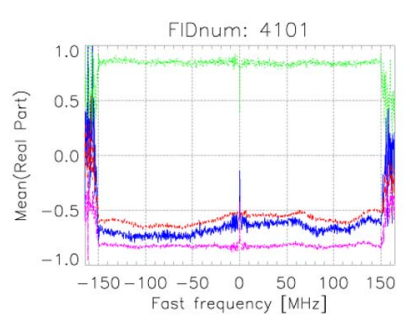

(a)

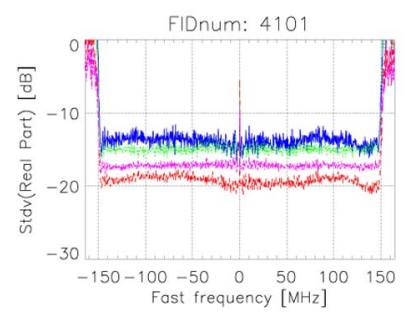

(c)

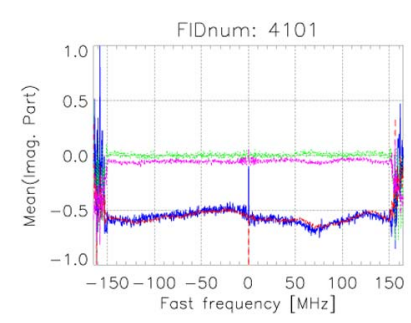

(b)

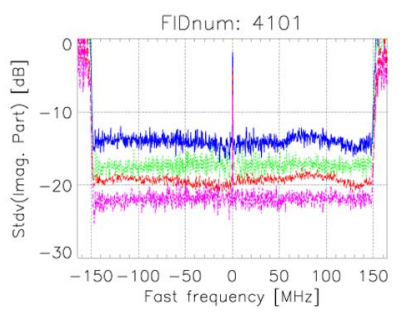

(d)
Fig. 12. Mean and standard deviations versus fast frequency for real and imaginary parts of the back-transformation matrix elements. $H_{11}^{-1}\left(f_{r}\right)$ : blue. $H_{12}^{-1}\left(f_{r}\right)$ : green. $H_{21}^{-1}\left(f_{r}\right)$ : red. $H_{22}^{-1}\left(f_{r}\right)$ : magenta.

fast-frequency samples. The estimated standard deviations for each fast-frequency bin are therefore relatively high. Decreased standard deviations could be achieved by averaging versus neighboring fast-frequency bins. However, averaging versus fast frequency reduces the resolution in fast frequency and should not be performed over fast-frequency intervals where the frequency response varies. In order to determine the fastfrequency intervals for which the transfer matrix elements can be assumed sufficiently constant, further data takes with an increased number of calibration pulses for each calibration beam are necessary. This will allow one to estimate the fore/aft to sum/difference channel transformation matrix with high accuracy and high fast-frequency resolution.

For stationary target signals after coregistration, the fore and aft channel signals should ideally be the same. Differences between the fore and aft channel data are called channel imbalances [24]. The channel imbalances $C\left(f_{r, k}, f_{l}\right)$ are defined in (24), shown at the bottom of the page, where $Z_{I, 1}\left(f_{r, k}, f_{l}\right)$ and $Z_{I, 2}\left(f_{r, k}, f_{l}\right)$ are the reconstructed and coregistered fore and aft channel data in fast-frequency and Doppler-frequency domain; $f_{r, k}$ are the fast frequency and $f_{l}$ the Doppler-frequency samples. In order to reduce the noise and enhance the image quality, a moving average filter is applied which averages over $2 N+1$ neighboring fast frequency and $2 M+1$ neighboring Dopplerfrequency samples. In Fig. 13(a) and (b), the channel imbalances are shown for reconstructing the fore and aft channel data under the assumption of ideal receive hardware. This means only the phase offset between the sum and difference channel

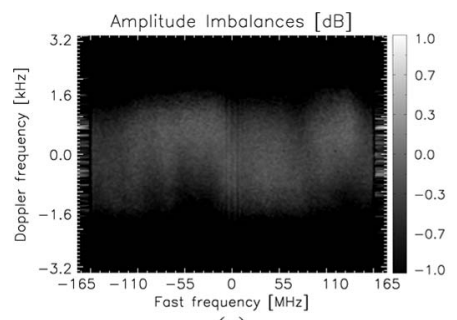

(a)

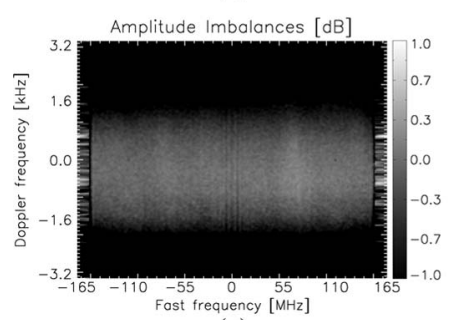

(c)

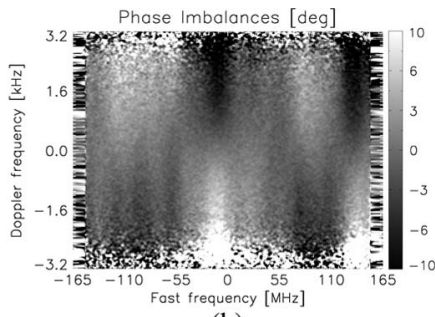

(b)

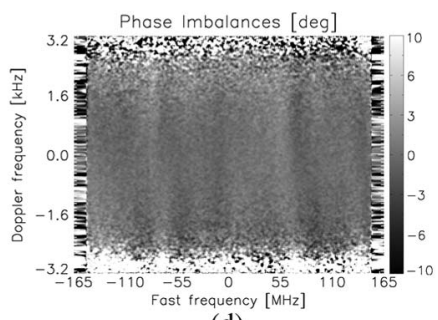

(d)
Fig. 13. Channel imbalances in (a) and (c) amplitude and (b) and (d) phase as a function of fast and Doppler frequency. In (a) and (b), the fore and aft channel data were reconstructed solely by phase offset compensation (simple model). In (c) and (d), the data were reconstructed by use of the complete estimated receive hardware transformation matrix as a function of fast frequency. $($ FIDnum $=4101)$.

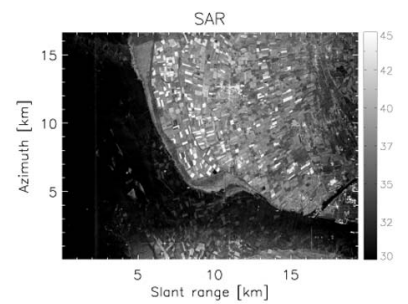

(a)

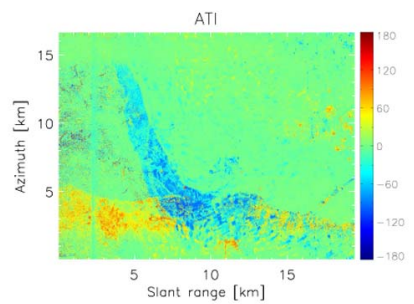

(b)
Fig. 14. (a) SAR image of reconstructed fore channel. (b) ATI phase of reconstructed fore and aft channel SAR images. The images show the mouth of the Elbe river $($ FIDnum $=9613)$.

data is compensated according to (9). In Fig. 13(c) and (d), the resulting channel imbalances are shown, when the receive hardware transformation matrix estimated as a function of fast frequency is applied for fore and aft channel reconstruction. Ideally, the amplitude imbalances should be $0 \mathrm{~dB}$ and the phase imbalances should be $0^{\circ}$. For the simple reconstruction algorithm, noticable imbalances in amplitude and phase can be observed which vary versus fast and Doppler frequency. On the other hand, a significant reduction in channel imbalances can be achieved by application of the proposed fore and aft channel reconstruction procedure.

\section{Examples on the GMTI Capability}

In Fig. 14(a), an example SAR image of a reconstructed fore channel can be seen, while the ATI image of the reconstructed

$$
C\left(f_{r, k}, f_{l}\right)=\frac{\sum_{n=k-N}^{k+N} \sum_{m=l-M}^{l+M} Z_{I, 1}\left(f_{r, k+n}, f_{l+m}\right) Z_{I, 2}^{\star}\left(f_{r, k+n}, f_{l+m}\right)}{\sum_{n=k-N}^{k+N} \sum_{m=l-M}^{l+M}\left|Z_{I, 1}\left(f_{r, k+n}, f_{l+m}\right)\right|^{2}}
$$


fore and aft channel signals after coregistration is shown in Fig. 14(b). For the reconstruction, the simple transformation matrix model according to Section II-D was used. The images show the mouth of the Elbe river in Northern Germany. In both images, azimuth ambiguities are visible in the water of the mouth of the Elbe river, because the calm water as the desired target has low SNR. This reveals the azimuth ambiguities which are usually not as clearly visible as in this case. While in the SAR image, the azimuth ambiguities appear as unexpected images of fields in the water, in the ATI image the azimuth ambiguities are characterized by their phase offset $\Delta \phi= \pm \pi d P R F / v$ caused by the back folding in Doppler frequency [15]. The images show that high-resolution fore and aft channel SAR images can be reconstructed by the proposed approach and that the proposed approach is able to restore the interferometric phase signal which is used in array processing techniques in order to suppress unwanted interference such as clutter suppression in the case of GMTI or azimuth ambiguity suppression for improved SAR imaging. A closer look at the ATI phase image allows one to identify some potential moving targets in the Elbe. In the next DRA mode campaign, data takes with ground truth will be available. This will allow for a detailed analysis of the GMTI performance of the reconstructed fore and aft channel DRA mode signals.

\section{Summary AND CONCLUSION}

In this paper, an approach for the fore and aft channel reconstruction in the TerraSAR-X DRA mode was developed. The proposed approach makes use of calibration pulses and calibration beams which are by default available for calibration purposes with TerraSAR-X. The theory for the compensation of the transmit and receive hardware impact and the reconstruction of the fore and aft channel signals in the case of the TerraSARX DRA mode by use of calibration pulses and calibration beams was developed and applied to experimental data. Experimental data evaluation showed that the fore and aft to sum and difference channel transformation is approximately an ideal hybrid coupler transformation followed by a phase offset which has an arbitrary but stable value during one data take. In the simple model, the phase offset varies from data take to data take. While a phase offset could be estimated solely from calibration pulses with CalDRA calibration beams, the estimation of the complete transformation matrix without an a priori model requires at least two different calibration beams, but yields a noticable improvement in the reduction of channel imbalances. Furthermore, the propagation of errors in the transformation matrix estimation due to $S N R$ limitations of the calibration pulses and $S N R$ limitations of the imaging data to the ATI signal of the reconstructed channels yields an explanation for the variations in along-track baseline estimation from data take to data take. In the next DRA mode campaign, data takes with a large number of calibration pulses will be employed in order to estimate the receive hardware transformation matrix with high $S N R$ and high fast-frequency resolution.

Although a multichannel implementation by use of sum and difference beams is used for modern tracking radars due to their affordability and is known as $\Sigma \Delta-S T A P$ [21], [26], there is no implementation of sum and difference channels for spaceborne SAR/GMTI. Furthermore, according to the authors' knowledge there is no publication on the increased complexity for the reconstruction of the fore and aft channel signals with highresolution SAR systems such as TerraSAR-X employing pulse bandwidths of up to $300 \mathrm{MHz}$ and coherent integration times over several thousand pulses. In this paper, it was shown for the first time that with the TerraSAR-X DRA mode high-resolution spaceborne SAR images of the fore and aft channels can be reconstructed and the along-track interferometric phase can be restored.

\section{ACKNOWLEDGMENT}

The authors would like to thank the TerraSAR-X team and, particularly, J. Mittermayer of the Microwaves and Radar Institute for providing the experimental TerraSAR-X processor, which gave us the opportunity to process and analyze the TerraSAR-X DRA mode raw data. The authors would also like to thank S. Suchandt and H. Runge of the Remote Sensing Technology Institute (IMF) for planning many of the experiments performed in the frame of the DRA mode campaigns, and A. Niedermeier of IMF for the fruitful discussions.

\section{REFERENCES}

[1] M. Suess, S. Riegger, W. Pitz, and R. R. Werninghaus, "TerraSARXDesign and performance," in Proc. EUSAR, Ulm, Germany, May 2004.

[2] J. Mittermayer and H. Runge, "Conceptual studies for exploiting the TerraSAR-X dual receive antenna," in Proc. IEEE IGARSS, Toulouse, France, Jul. 2003, pp. 2140-2142.

[3] P. Meisl, A. A. Thompson, and A. P. Luscombe, "Radarsat-2 mission: Overview and development status," in Proc. ACRS, Taipei, Taiwan, Dec. 2000, pp. 373-376.

[4] S. Mezzasoma, A. Gallon, F. Impagnatiello, G. Angino, S. Fagioli, A. Capuzi, F. Caltagirone, R. Leonardi, and U. Ziliotto, "COSMOSkyMed system commissioning: End-to-end system performance verification," in Proc. IEEE RADAR, Rome, Italy, May 2008, pp. 1092-1096.

[5] H. Runge, S. Suchandt, A. Kotenkov, H. M. Vonavka, and U. Balss, "Space borne SAR traffic monitoring," in Proc. IRS, Cologne, Germany, Sep. 2007.

[6] A. Gabban, H. Greidanus, A. J. E. Smith, L. Anitori, F.-X. Thoorens, and J. Mallorqui, "Ship surveillance with TerraSAR-X ScanSAR," in Proc. 3rd TerraSAR-X Sci. Team Meeting, Oberpfaffenhofen, Germany, Nov. 2008.

[7] R. Siegmund, M. Bao, S. Lehner, A. Niedermeier, and R. Mayerle, "Surface currents imaged with hybrid along and cross track interferometry," in Proc. IEEE IGARSS, Sydney, Australia, Jul. 2001, pp. 3146-3148.

[8] R. Romeiser, H. Runge, S. Suchandt, J. Sprenger, H. Weilbeer, A. Sohrmann, and D. Stammer, "Current measurements in rivers by spaceborne along-track InSAR," IEEE Trans. Geosci. Remote Sens., vol. 45, no. 12, pp. 4019-4031, Dec. 2007.

[9] J. Schulz-Stellenfleth, S. Lehner, and J. Horstmann, "Use of TerraSARX for oceanography," in Proc. IEEE IGARSS, Seoul, Korea, Jul. 2005, pp. $4886-4889$

[10] I. Hajnsek and K. Papathanassiou, "Exploring the polarimetric modes of TerraSAR-X for quantitative parameter estimation," in Proc. IEEE IGARSS, Boston, MA, Jul. 2008.

[11] D. M. Pozar, Microwave Engineering, 3rd ed. New York: Wiley, 2005.

[12] P. D. Beaulne, C. H. Gierull, C. E. Livingstone, I. C. Sikaneta, S. Chiu, S. Gong, and M. Quinton, "Preliminary design of a SAR/GMTI processing system for RADARSAT-2 MODEX data," in Proc. IEEE IGARSS, Toulouse, France, Jul. 2003, pp. 1019-1021.

[13] M. Schwerdt, D. Hounam, B. Bräutigam, and J. L. Alvarez-Perez, "TerraSAR-X: Calibration concept of a multiple mode high resolution SAR," in Proc. IEEE IGARSS, Seoul, Korea, Jul. 2005, pp. 4874-4877.

[14] A. Moreira, J. Mittermayer, and R. Scheiber, "Extended chirp scaling algorithm for air- and spaceborne SAR data processing in stripmap and scanSAR imaging modes," IEEE Trans. Geosci. Remote Sens., vol. 34, no. 5, pp. 1123-1136, Sep. 1996 
[15] M. Gabele and I. Sikaneta, "Motion parameter estimation of Doppler ambiguous moving targets in SAR-GMTI," in Proc. IRS, Cologne, Germany, Sep. 2007.

[16] M. Schwerdt, B. Bräutigam, M. Bachmann, B. Döring, D. Schrank, and J. Hueso Gonzalez, "TerraSAR-X calibration results," in Proc. IEEE IGARSS, Boston, MA, Jul. 2008, pp. II-205-II-208.

[17] M. Stangl, R. Werninghaus, B. Schweizer, C. Fischer, M. Brandfass, J. Mittermayer, and H. Breit, "TerraSAR-X technologies and first results," IEEE Trans. Antennas Propag., vol. 153, no. 2, pp. 86-95, Apr. 2006.

[18] A. V. Oppenheim, R. W. Schäfer, and J. R. Buck, Discrete-Time Signal Processing, 2nd ed. Englewood Cliffs, NJ: Prentice-Hall, 2005.

[19] L. L. Scharf, Statistical Signal Processing: Detection, Estimation, and Time Series Analysis. Addison-Wesley, 1991.

[20] M. Schwerdt, B. Bräutigam, and M. Bachmann, "TerraSAR-X instrument and operations and calibration segment," Microwaves Radar Inst., German Aerosp. Center (DLR), Issue 1.5, Tech. Note TSX-IOCS-DD4311, Feb. 2007

[21] R. Klemm, Principles of Space-Time Adaptive Processing, 2nd ed. London, U.K.: IEE, 2002.

[22] W.-D. Wirth, Radar Techniques Using Array Antennas. London, U.K.: IEE, 2001

[23] W. Hackbusch, H. R. Schwarz, and E. Zeidler, Teubner Taschenbuch der Mathematik. Wiesbaden, Germany: B.G. Teubner Verlag, 1996.

[24] C. H. Gierull, "Digital channel balancing of along-track interferometric SAR data," DREO, Ottawa, ON, Canada, Mar. 2003.

[25] B. Schättler, "Datatake file handling and identification," Appl. Remote Sens. Cluster, German Aerosp. Center (DLR), Cologne, Germany, Oct. 2008.

[26] R. D. Brown, R. A. Schneible, M. C. Wicks, H. Wang, and Y. Zhang, "STAP for clutter suppression with sum and difference beams," IEEE Trans. Aerosp. Electron. Syst., vol. 36, no. 2, pp. 634-646, Apr. 2000.

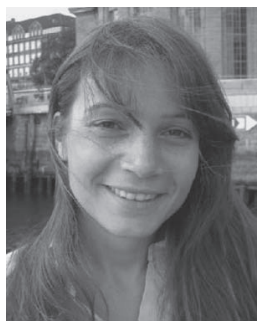

Martina Gabele received the Dipl.Ing. degree in electrical engineering from the Universität Karlsruhe (TH), Karlsruhe, Germany, in 2004. She is currently working toward the Ph.D. degree in the Microwaves and Radar Institute, German Aerospace Center (DLR), Wessling, Germany, focusing on the area of system concepts and processing methods for spaceborne synthetic aperture radar (SAR) imaging and ground moving target indication (GMTI).

Since 2004, she has been with the New SAR Missions Group and, since 2008, with the SAR Techniques Group of the Microwaves and Radar Institute, DLR. In 2006/2007, she was a Visiting Scientist with the Radar Systems Section of Defense R\&D Canada (DRDC), Ottawa, ON, Canada.

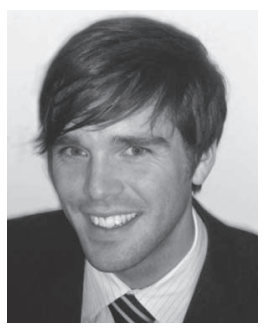

Benjamin Bräutigam received the Dipl.Ing. degree in electrical engineering from the Universität Karlsruhe (TH), Karlsruhe, Germany, in 2003.

In 2003, he was a Visiting Scientist with the National Oceanic and Atmospheric Administration's Environmental Technology Laboratory, Boulder, CO. In 2004, he joined the Microwaves and Radar Institute, German Aerospace Center (DLR), Wessling, Germany. He was responsible for internal instrument calibration in spaceborne synthetic aperture radar projects like TerraSAR-X, TerraSAR-L, TanDEM-X, and Global Monitoring for Environment and Security Sentinel-1. Since 2008, he has been the Head of the System Performance Group, working on TerraSAR-X and TanDEM-X. His major research interests include the development and analysis of innovative methods for instrument calibration and performance monitoring.

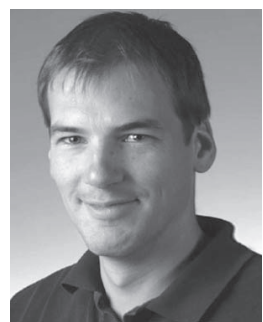

Daniel Schulze received the Diploma degree in aerospace technology from the University of Berlin, Berlin, Germany, in 2002.

Since 2004, he has been with the Microwaves and Radar Institute, German Aerospace Center (DLR), Wessling, Germany, in the system engineering and calibration segment (SEC) of TerraSAR-X. In the years 2004 and 2005, he implemented the Instrument Command Generator and supervised the Long-Term Database implementation. Later on, he was a System Engineer for the Instrument Operations and Calibration Segment (IOCS), which are the operational systems of the SEC. During the test and integration phase, he coordinated the IOCS-internal and the IOCSground segment tests. Since 2008, he has been the Project Manager of the joined TerraSAR-X and TanDEM-X SEC.

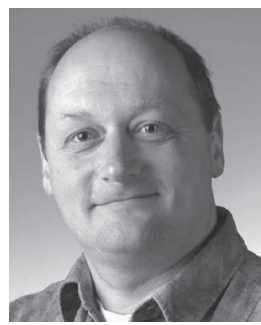

Ulrich Steinbrecher received the Dipl.Ing. degree in electrical engineering/communication from the University of Siegen, Siegen, Germany, in 1990.

In 1990, he started his work with the German Aerospace Center (DLR), Wessling, Germany, with the development of a synthetic aperture radar (SAR) raw data simulator. Then, he worked in software development for the X-band SAR (X-SAR) processor for the joint U.S.-Italian-German SIR-C/X-SAR missions in 1994. When the data were in-housed, he concentrated on aspects of operational SAR processing of high data volumes. In 1995, he pioneered a completely automatic SAR processing system based on a robot-maintained mass memory archive. Before he became responsible for the development of the raw data analysis and screening system of the Shuttle Radar Topography Mission (SRTM), he developed the software for a phase-preserving ScanSAR processor for Radarsat-1. In the time between the SRTM mission and the start of the TerraSAR-X project, he left for two years the SAR domain and worked for the SCIAMACHY LIMB processor. Since 2002, he has been concerned with the TerraSAR-X radar system, and since the launch in 2007 , he is responsible for TerraSAR-X SAR instrument operations. He is currently with the Microwaves and Radar Institute, DLR.

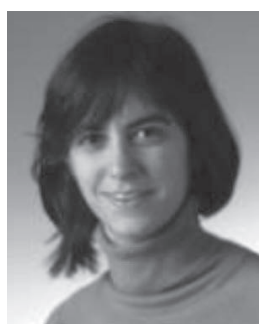

Nuria Tous-Ramon received the Telecommunications Engineering degree from the Universitat Politècnica de Catalunya, Barcelona, Spain, and the Double Diploma degree in electrical engineering from the Technical University of Darmstadt, Darmstadt, Germany, in 2006.

In 2006, she started working with the Microwaves and Radar Institute, German Aerospace Center (DLR), Wessling, Germany, in the TerraSAR-X System Engineering and Calibration Group (SEC). She supported the launch and successful Commissioning Phase of TerraSAR-X and is part of the Synthetic Aperture Radar (SAR) Instrument Operations Team for the TerraSAR-X and TanDEM-X Projects. Since the end of 2007 until the beginning of 2009, she also was with the TerraSAR-X experimental SAR processor with the SAR System Engineering Group, Microwaves and Radar Institute.

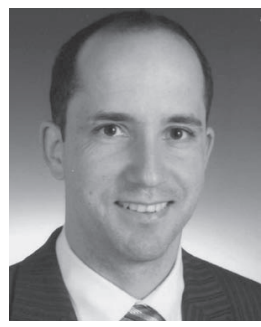

Marwan Younis (S'95-M'05-SM'08) was born in Las Cruces, NM, in 1970. He received the B.Sc. degree in electrical engineering from the University of Baghdad, Baghdad, Iraq, in 1992 and the Dipl.Ing. (M.Sc.) and Dr.Ing. (Ph.D.) degrees in electrical engineering from the Universität Karlsruhe (TH), Karlsruhe, Germany, in 1997 and 2004, respectively.

From 1998 to 2004, he was a Research Scientist with the Institut für Höchstfrequenztechnik und Elektronik, TH. Since 2005, he has been with the Microwaves and Radar Institute, German Aerospace Center (DLR), Wessling, Germany. He is the author or coauthor of over 40 conference papers and more than 15 reviewed publications. His research fields include synthetic aperture radar (SAR) systems, digital beamforming for radar, synchronization of bistatic SAR, forward-looking radar, and antennas.

Dr. Younis is an active member of the German Association for Location and Navigation. He is working group leader within the IEEE Instruments and Future Technologies Technical Committee. He is a Lecturer with TH. He received the Hermann-Billing award for his Ph.D. thesis in 2005. 